\title{
Optical photometry and spectral classification in the field of the open cluster NGC 6996 in the North America Nebula ${ }^{\star \star \star}$
}

\author{
S. Villanova ${ }^{1}$, G. Baume ${ }^{1,2}$, G. Carraro ${ }^{1}$, and A. Geminale ${ }^{1}$ \\ 1 Dipartimento di Astronomia, Università di Padova, Vicolo Osservatorio 2, 35122 Padova, Italy \\ 2 Facultad de Ciencias Astronómicas y Geofísicas de la UNLP, IALP-CONICET, Paseo del Bosque s/n, La Plata, Argentina
}

Received 7 November 2003 / Accepted 30 January 2004

\begin{abstract}
We present and discuss broad band $\mathrm{CCD} U B V(I)_{\mathrm{C}}$ photometry and low resolution spectroscopy for stars in the region of the open cluster NGC 6996, located in the North America Nebula. The new data allow us to tightly constrain the basic properties of this object. We revise the cluster size, which in the past has been significantly underestimated. The width of the Main Sequence is mainly interpreted in terms of differential reddening, and indeed the stars' color excess $E_{B-V}$ ranges from 0.43 to 0.65 , implying the presence of a significant and evenly distributed dust component. We cross-correlate our optical photometry with near infrared photometry from 2MASS, and by means of spectral classification we are able to build extinction curves for an handful of bright members. We find that the reddening slope and the total to selective absorption ratio $R_{V}$ toward NGC 6996 are anomalous. Moreover the reddening-corrected colors and magnitudes allow us to derive estimates for the cluster distance and age, which turn out to be $760 \pm 70 \mathrm{pc}\left(V_{0}-M_{V}=9.4 \pm 0.2\right)$ and $\sim 350 \mathrm{Myr}$, respectively. Based on our results, we suggest that NGC 6996 is located in front of the North America Nebula, and does not seem to have any apparent relationship with it.
\end{abstract}

Key words. Galaxy: open clusters and associations: individual: NGC 6996 - Galaxy: open clusters and associations: general

\section{Introduction}

Galactic open clusters are fundamental tools to probe the global properties and evolution of the Galactic disk. The determination of their fundamental parameters (age, distance, reddening pattern, size) allows us not only to better understand the properties and evolution of the Galactic open cluster system as a whole, but in many cases to put constrains on large structures like super-clusters, complexes, HII regions and molecular clouds systems, which they might be part of or not.

This is the case of NGC $6996=\mathrm{C} 2054+444\left(l=85.47^{\circ}\right.$, $\left.b=-0.48^{\circ}\right)$, which is believed to lie in front of the North America Nebula (NGC 7000) HII region, near its western edge (see Fig. 1), where an important dust cloud separates this nebula from the nearby Pelican Nebula.

NGC 6996 is a sparse and moderately young open cluster $\left(\sim 10^{8}\right.$ Myr, Zdanavicius \& Straizys 1990). However, its basic parameters, in particular distance and reddening, are not well known, and this was the main motivation driving this study.

Send offprint requests to: G. Carraro, e-mail: giovanni.carraro@unipd.it

* Based on observations carried out at Asiago and Teramo Observatories, Italy.

$\star \star$ Photometry is only available in electronic form at the CDS via anonymous ftp to cdsarc.u-strasbg.fr $(130.79 .128 .5)$ or via http://cdsweb.u-strasbg.fr/cgi-bin/qcat?J/A+A/419/149
Previous investigations carried out in this area include the photographic studies by Muller (1936) and Barkhatova (1958). Later, a more detailed study was conducted by Zdanavicius \& Straizys (1990), who obtained photoelectric measurements of several bright stars in the region of NGC 6996 in the Vilnius system. More recently, Subramaniam et al. (1995) catalogued this cluster as a probable binary together with the twin cluster Collinder 428. This latter object is located in the opposite (the eastern) border of the North America Nebula, and indeed both clusters might be related to the HII region. The entire area belongs to the Cygnus region, which has been studied rather intensively.

This region (from $l \sim 74^{\circ}$ to $l \sim 85^{\circ}$ ) has the singular property that stars present an anomalous interstellar reddening law, with a larger slope (see Straizys et al. 1999 and references therein). In particular, the visual absorption distribution in this region was studied by Goudis \& White (1979) with the $\mathrm{H}_{\alpha}$ surface brightness technique, by Bally \& Scoville (1980) by using ${ }^{12} \mathrm{CO}$ observations and, finally, by Cambrésy et al. (2002) from 2MASS data. These latter authors discovered some new possible star clusters toward this area, which might be physical objects and as a consequence deserve further investigation.

To derive better estimates of the NGC 6996 basic parameters (reddening, distance and age), to study the reddening law in this region and to understand whether some relation exists between the cluster and the North America Nebula, we performed 


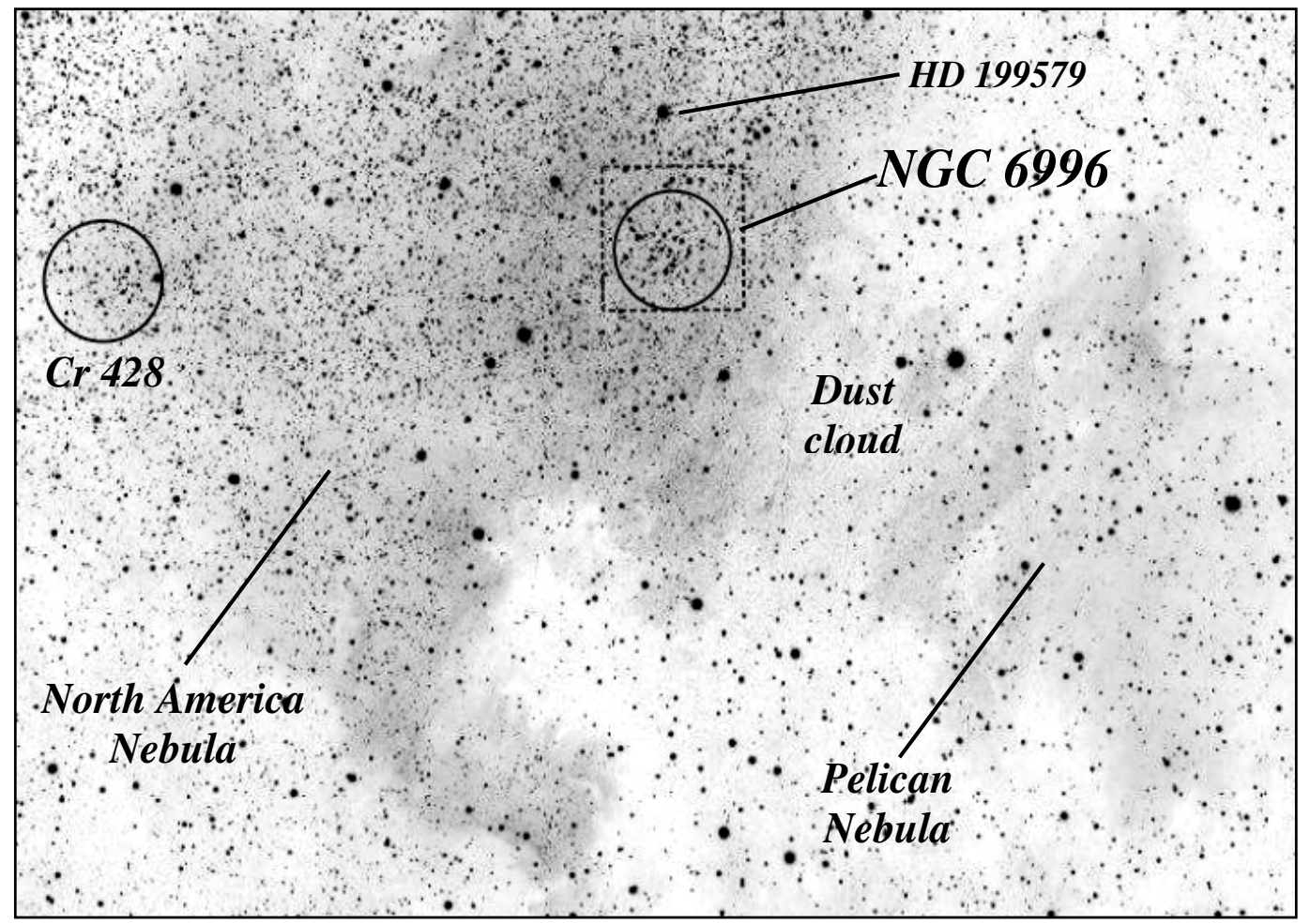

Fig. 1. A map of the North America Nebula region. The region of NGC 6996 covered by our study is marked by a dashed square (see also Fig. 3). North is up, East to the left.

$\mathrm{CCD} U B V(I)_{\mathrm{C}}$ photometry covering most of the cluster region, and complement it with low resolution spectroscopic observation of bright stars in this region. Our data were also crosscorrelated with near infrared $\left(J H K_{\mathrm{S}}\right)$ data from the 2MASS catalogue and with astrometric information available from the Tycho-2 catalogue (Høg et al. 2000) for the brightest stars.

The layout of the paper is as follows. In Sect. 2 we describe our observations and the reduction procedure. In Sect. 3 we present the data analysis. Section 4 deals with the cluster basic parameters determination. Section 5 is devoted to a brief discussion of the outcome of this study.

\section{Observations and data reduction}

\subsection{Photometry}

CCD $U B V(I)_{\mathrm{C}}$ data were obtained during three observational runs. Two of them were carried out with the AFOSC camera at the $1.82 \mathrm{~m}$ Copernico telescope of Cima Ekar (Asiago, Italy), on the photometric nights of November 8, 2002 and September 18, 2003. AFOSC samples a 8.14 × 8.14 field in a $1 \mathrm{~K} \times 1 \mathrm{~K}$ nitrogen-cooled thinned $\mathrm{CCD}$ and this camera was used to take a central and a south frame in the cluster area. The other run was conducted at Teramo Observatory (Italy) using a $512 \times 512 \mathrm{CCD}$ onboard the $0.72 \mathrm{~m}$ Teramo Normale Telescope (TNT). Teramo observations were used to complement Asiago ones since two peripheral and less deep fields were taken during a non-photometric night. Figures 1 and 3 show the finding charts of the covered area.

Details of the observations are listed in Table 1, where the observed fields are reported together with the exposure
Table 1. Journal of observations of NGC 6996 and standard star fields together with calibration coefficients.

\begin{tabular}{|c|c|c|c|c|c|c|}
\hline Field & Filter & \multicolumn{3}{|c|}{$\begin{array}{c}\text { Exposure time } \\
{[\mathrm{s}]}\end{array}$} & \multirow{2}{*}{ 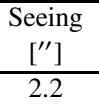 } & \multirow{2}{*}{$\begin{array}{c}\text { Air-mass } \\
1.091\end{array}$} \\
\hline NGC 6996 & $U$ & $900 \times 2$ & 180 & 10 & & \\
\hline 1 frame & $B$ & 600 & 60 & 10 & 2.1 & 1.062 \\
\hline Asiago & $V$ & 300 & 30 & 5 & 2.2 & 1.052 \\
\hline $8 / 11 / 02$ & $I$ & 300 & 30 & 5 & 2.3 & 1.045 \\
\hline NGC 6996 & $U$ & 900 & - & - & $\sim 3$ & 1.368 \\
\hline 2 frames & $B$ & 600 & - & - & $\sim 3$ & 1.322 \\
\hline Teramo & $V$ & 300 & 60 & - & $\sim 3$ & 1.251 \\
\hline $5-12-2002$ & $I$ & 180 & 60 & - & $\sim 3$ & 1.219 \\
\hline NGC 6996 & $U$ & 1200 & 120 & - & 1.9 & 1.007 \\
\hline 1 frame & $B$ & 800 & 80 & 8 & 1.8 & 1.018 \\
\hline Asiago & $V$ & 600 & 60 & 6 & 1.8 & 1.027 \\
\hline $18 / 09 / 03$ & $I$ & 400 & 40 & 4 & 1.7 & 1.037 \\
\hline PG 0231+051 & $\bar{U}$ & 800 & & & 2.5 & 1.348 \\
\hline Asiago & $B$ & 300 & & & 2.4 & 1.324 \\
\hline $8 / 11 / 02$ & $V$ & 60 & & & 2.2 & 1.316 \\
\hline & $I$ & 90 & & & 2.2 & 1.315 \\
\hline PG 2213-006 & $U$ & 600 & & & 2.5 & 1.447 \\
\hline Asiago & $B$ & 150 & & & 2.3 & 1.457 \\
\hline $8 / 11 / 02$ & $V$ & 30 & & & 2.3 & 1.465 \\
\hline & $I$ & 30 & & & 2.3 & 1.472 \\
\hline Calibration & $u_{1}=+$ & $861 \pm 0$. & & & $+1.602 \pm$ & .004 \\
\hline coefficients & $u_{2}=-$ & $142 \pm 0$ & & $b_{2}$ & $+0.038 \pm$ & .006 \\
\hline Asiago & $u_{3}=+$ & & & $b_{3}$ & +0.29 & \\
\hline $8 / 11 / 02$ & $v_{1 b v}=$ & $1.003 \pm 0$ & & $i_{1}=$ & $+1.691 \pm$ & 044 \\
\hline & $v_{2 b v}=$ & $0.016 \pm 0$ & & $i_{2}=$ & $+0.057 \pm$ & .043 \\
\hline & $v_{3}=+$ & & & & & \\
\hline & $v_{1 v i}=$ & $.002 \pm 0$ & & & & \\
\hline & $v_{2 v i}=$ & $.013 \pm 0$ & & & & \\
\hline
\end{tabular}




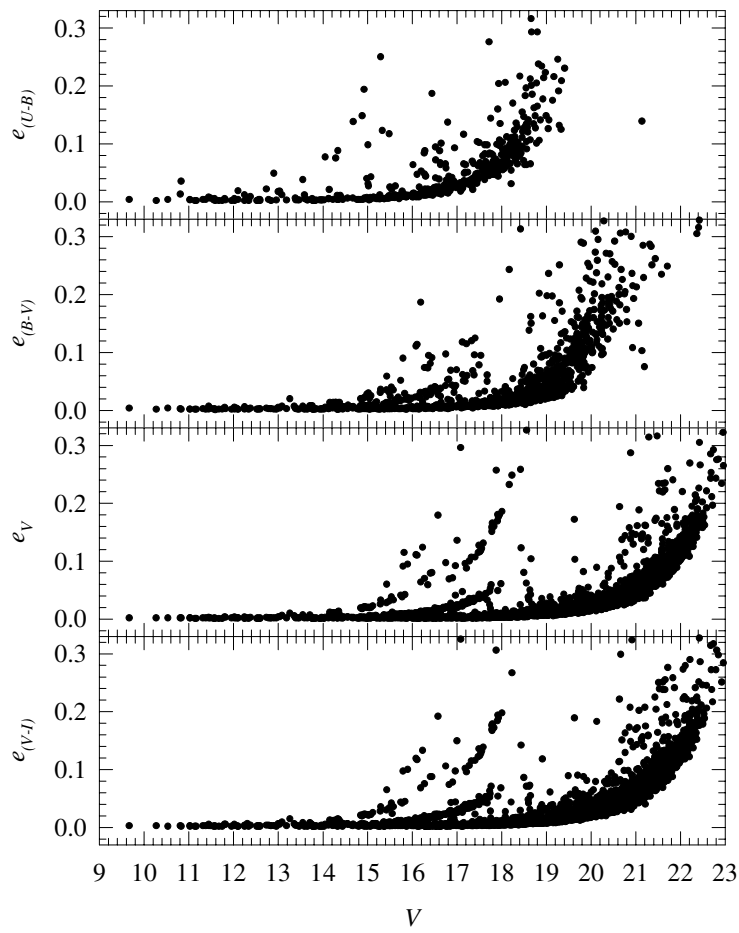

Fig. 2. DAOPHOT errors in the colour indexes and $V$ magnitude as a function of $V$.

times, the typical seeing values and the air-masses. The data were reduced with the IRAF ${ }^{1}$ packages CCDRED, DAOPHOT, and PHOTCAL using the point spread function (PSF) method (Stetson 1987) for the frame obtained on 8/11/02 (Asiago) and using only aperture photometry for the others. The calibration equations obtained by observing Landolt (1992) PG 0231+051 and PG 2213-006 fields at the Asiago Observatory, are:

$$
\begin{aligned}
u & =U+u_{1}+u_{2}(U-B)+u_{3} X \\
b & =B+b_{1}+b_{2}(B-V)+b_{3} X \\
v & =V+v_{1 b v}+v_{2 b v}(B-V)+v_{3} X \\
v & =V+v_{1 v i}+v_{2 v i}(V-I)+v_{3} X \\
i & =I+i_{1}+i_{2}(V-I)+i_{3} X
\end{aligned}
$$

where $U B V I$ are standard magnitudes, $u b v i$ are the instrumental ones, $X$ is the air-mass and the derived coefficients are presented in the bottom of Table 1 . Since observations from Teramo Observatory were taken during a non photometric night, they were zero-point and color-term related to the Asiago data. $V$ magnitudes, when the $B$ magnitude was available, we used expression (3) to compute them, elsewhere expression (4) was used. The standard stars in these fields provide a very good color coverage which allows us to obtain reliables transformations. For the extinction coefficients, we assumed the typical values for the Asiago Observatory (Desidera et al. 2002 ${ }^{2}$ ). The photometric error trends against the $V$ magnitude data are shown in Fig. 2, where one can clearly distinguish the error trends for TNT and Asiago observations.

\footnotetext{
1 IRAF is distributed by NOAO, which are operated by AURA under cooperative agreement with the NSF.

2 www.pd.astro.it/Asiago/5000/5100/5100.html
}

The photometric data for some of the brightest stars in the region of NGC 6996 are shown in Table 2. The full table is only available electronically at CDS.

\subsection{Spectroscopy}

Spectroscopic observations were carried out with the AFOSC camera at the $1.82 \mathrm{~m}$ Copernico telescope of Cima Ekar (Asiago, Italy), on nights of July 21, September 18 and October 1, 2003. The instrument was used in low dispersion mode $(R=600)$, the grism \#4 was chosen to have a large spectral coverage (3500-7000 A) and the exposure time ranged from 10 to $30 \mathrm{~min}$, according to the brightness of the stars. The data has been reduced with the IRAF package for onedimensional spectroscopy CTIOSLIT and by using the $\mathrm{Hg}-\mathrm{Cd}$ lamp spectra for wavelength calibration purposes. To have fluxcalibration, a few standard spectrophotometric stars were also observed. Spectra (see Fig. 4) were classified in two different ways:

- by comparing them with a library of spectroscopic standards (e.g. Jacoby et al. 1984; Torres-Dodgen \& Weawer 1993);

- by measuring the equivalent width of some absorption lines (mainly $\mathrm{H}_{\alpha}, \mathrm{H}_{\beta}$ and $\mathrm{H}_{\gamma}$ ) (see Jaschek \& Jaschek 1987).

At this resolution we estimate an error in the spectral type derivation of \pm 1 tenth. The derived spectral types and luminosity classes are reported in Table 2 and Fig. 4.

\section{Data analysis}

\subsection{Cluster angular size}

To derive an estimate of the cluster angular size, we computed surface stellar densities at increasing concentric $1^{\prime}$ wide annuli around the adopted cluster center $\left(\alpha_{2000}=20: 56: 30 ; \delta_{2000}=\right.$ $+44: 38: 00)$ over: $a$ ) the corresponding DSS $-2^{3}$ red image, and $b$ ) the 2MASS infrared data. The results are shown in Fig. 5, where the dotted line is the field star density level as derived from mean star counts in two 2MASS fields, 20' southward and northward NGC 6996, respectively. The patchy distribution of dust and gas clearly renders it difficult to fix a cluster radius. However, by inspecting both DSS maps and this plot we argue that the cluster radius is $\sim 7^{\prime}$, the point (dashed vertical line in Fig. 5) at which the stellar density more clearly reaches the field level. Therefore, we adopt this value as angular radius, which turns out to be almost twice the estimates reported by Lyngå (1987) and Dias et al. (2002) (6' and $8^{\prime}$ in diameter, respectively).

\subsection{Proper motions}

Important information on the kinematics and membership of the brightest stars in and around a star cluster might in principle be derived from the proper motions as available in the Tycho-2

\footnotetext{
${ }^{3}$ Second generation Digitized Sky Survey, http://cadcwww.dao.nrc.ca/cadcbin/getdss
} 
Table 2. Brightest stars in the region of NGC 6996.

\begin{tabular}{|c|c|c|c|c|c|c|c|c|c|c|}
\hline \# & $\#_{\mathrm{ZS}}$ & $\begin{array}{l}\text { 2MASS ID. } \\
\text { Tycho-2 ID. } \\
\text { BD ID. }\end{array}$ & $\begin{array}{l}X\left[{ }^{\prime \prime}\right] \\
Y\left[{ }^{\prime \prime}\right]\end{array}$ & $\begin{array}{l}\alpha_{2000} \\
\delta_{2000}\end{array}$ & $\bar{V}$ & $\begin{array}{l}B-V \\
U-B \\
V-I\end{array}$ & $\begin{array}{l}E_{B-V} \\
E_{U-B} \\
E_{V-I}\end{array}$ & $\begin{array}{c}\mu_{\alpha} \cos (\delta)[\mathrm{mas} / \mathrm{yr}] \\
\mu_{\delta}[\mathrm{mas} / \mathrm{yr}]\end{array}$ & $\overline{S T}$ & Memb. \\
\hline 2 & 26 & $\begin{array}{l}\text { J20563677+443538 } \\
\text { TYC 3179-687-1 } \\
\text { BD +44 3638 }\end{array}$ & $\begin{array}{r}-74.6 \\
-141.5\end{array}$ & $\begin{array}{l}20: 56: 36.9 \\
44: 35: 37.9\end{array}$ & 10.27 & $\begin{array}{c}0.35 \\
-0.08 \\
0.24\end{array}$ & $\begin{array}{c}0.15 \\
-0.18\end{array}$ & $\begin{array}{l}4.1 \pm 1.4 \\
6.1 \pm 1.4\end{array}$ & A7 V & $n m$ \\
\hline 13 & $50^{*}$ & $\begin{array}{l}\text { J20570129+444213 } \\
\text { TYC 3179-658-1 }\end{array}$ & $\begin{array}{r}-332.6 \\
254.7\end{array}$ & $\begin{array}{l}20: 57: 01.2 \\
44: 42: 13.2\end{array}$ & 11.44 & $\begin{array}{l}0.64 \\
0.40 \\
0.89\end{array}$ & $\begin{array}{l}0.39 \\
0.30\end{array}$ & $\begin{array}{l}-2.8 \pm 2.3 \\
-8.6 \pm 2.2\end{array}$ & A8 III & $n m$ \\
\hline 14 & 47 & $\begin{array}{l}\text { J20565989+444058 } \\
\text { TYC 3179-805-1 }\end{array}$ & $\begin{array}{r}-318.3 \\
179.9\end{array}$ & $\begin{array}{l}20: 56: 59.9 \\
44: 40: 58.3\end{array}$ & 11.48 & $\begin{array}{l}0.45 \\
0.35 \\
0.66\end{array}$ & $\begin{array}{l}0.44 \\
0.33 \\
0.64\end{array}$ & $\begin{array}{l}-3.5 \pm 2.3 \\
-7.8 \pm 2.3\end{array}$ & $\mathrm{~A} 1 \mathrm{~V}$ & $\operatorname{lm}$ \\
\hline 15 & 14 & J20562963+443939 & $\begin{array}{r}4.1 \\
99.1\end{array}$ & $\begin{array}{l}20: 56: 29.6 \\
44: 39: 39.2\end{array}$ & 11.56 & $\begin{array}{l}1.44 \\
1.33 \\
1.74\end{array}$ & $\begin{array}{l}0.52 \\
0.44\end{array}$ & & K2 III & pm \\
\hline 17 & 21 & $\begin{array}{l}\text { J20563310+443810 } \\
\text { TYC 3179-87-1 }\end{array}$ & $\begin{array}{r}-33.4 \\
11.0\end{array}$ & $\begin{array}{l}20: 56: 33.1 \\
44: 38: 10.7\end{array}$ & 11.60 & $\begin{array}{l}0.52 \\
0.45 \\
0.81\end{array}$ & $\begin{array}{l}0.44 \\
0.37 \\
0.72\end{array}$ & $\begin{array}{l}-3.7 \pm 2.3 \\
-8.9 \pm 2.1\end{array}$ & $\mathrm{~A} 3 \mathrm{~V}$ & $\operatorname{lm}$ \\
\hline 23 & 41 & $\mathrm{~J} 20565330+443422$ & $\begin{array}{l}-250.4 \\
-216.5\end{array}$ & $\begin{array}{l}20: 56: 53.3 \\
44: 34: 21.9\end{array}$ & 12.02 & $\begin{array}{l}0.51 \\
0.33 \\
0.83\end{array}$ & $\begin{array}{l}0.58 \\
0.53 \\
0.89\end{array}$ & & B9 V & $\operatorname{lm}$ \\
\hline 24 & $37^{*}$ & J20564701+443725 & $\begin{array}{r}-182.1 \\
-33.6\end{array}$ & $\begin{array}{l}20: 56: 47.0 \\
44: 37: 25.4\end{array}$ & 12.02 & $\begin{array}{l}0.41 \\
0.32 \\
0.57\end{array}$ & $\begin{array}{l}0.24 \\
0.22\end{array}$ & & $\mathrm{~A} 6 \mathrm{~V}$ & $n m$ \\
\hline 27 & 34 & J20564536+443523 & $\begin{array}{l}-165.8 \\
-155.6\end{array}$ & $\begin{array}{l}20: 56: 45.4 \\
44: 35: 23.4\end{array}$ & 12.10 & $\begin{array}{l}0.57 \\
0.41 \\
1.05\end{array}$ & $\begin{array}{l}0.59 \\
0.43 \\
1.06\end{array}$ & & $\mathrm{~A} 0 \mathrm{~V}$ & $\operatorname{lm}$ \\
\hline 28 & $48^{*}$ & $\mathrm{~J} 20570072+443503$ & $\begin{array}{l}-329.3 \\
-174.7\end{array}$ & $\begin{array}{l}20: 57: 00.7 \\
44: 35: 03.4\end{array}$ & 12.25 & $\begin{array}{l}0.53 \\
0.44 \\
0.86\end{array}$ & $\begin{array}{l}0.52 \\
0.42 \\
0.84\end{array}$ & & $\mathrm{~A} 1 \mathrm{~V}$ & $\operatorname{lm}$ \\
\hline 29 & 5 & $\begin{array}{l}\text { J20562065+443811 } \\
\text { TYC 3179-81-1 }\end{array}$ & $\begin{array}{l}99.9 \\
11.4\end{array}$ & $\begin{array}{l}20: 56: 20.6 \\
44: 38: 11.9\end{array}$ & 12.25 & $\begin{array}{l}0.56 \\
0.49 \\
0.95\end{array}$ & $\begin{array}{l}0.48 \\
0.41 \\
0.86\end{array}$ & $\begin{array}{l}-1.4 \pm 2.0 \\
-8.4 \pm 1.9\end{array}$ & A3 V & $\operatorname{lm}$ \\
\hline 31 & 2 & J20561119+443817 & $\begin{array}{r}200.2 \\
16.4\end{array}$ & $\begin{array}{l}20: 56: 11.2 \\
44: 38: 17.4\end{array}$ & 12.31 & $\begin{array}{l}1.42 \\
2.94 \\
2.40\end{array}$ & 0.38 & & $\mathrm{~K} 4 \mathrm{~V}$ & $n m$ \\
\hline 32 & 15 & J20563011+443704 & $\begin{array}{r}-1.8 \\
-55.6\end{array}$ & $\begin{array}{l}20: 56: 30.1 \\
44: 37: 04.3\end{array}$ & 12.34 & $\begin{array}{l}0.57 \\
0.46 \\
0.97\end{array}$ & $\begin{array}{l}0.59 \\
0.48 \\
0.98\end{array}$ & & $\mathrm{~A} 0 \mathrm{~V}$ & $l m$ \\
\hline 34 & 8 & $\mathrm{~J} 20562200+443537$ & $\begin{array}{r}84.8 \\
-144.7\end{array}$ & $\begin{array}{l}20: 56: 21.9 \\
44: 35: 35.5\end{array}$ & 12.52 & $\begin{array}{l}0.73 \\
0.38 \\
0.56\end{array}$ & $\begin{array}{l}0.72 \\
0.36\end{array}$ & & $\mathrm{~A} 1 \mathrm{~V}$ & $n m$ \\
\hline 36 & 30 & $\mathrm{~J} 20564188+444113$ & $\begin{array}{r}-125.9 \\
194.1\end{array}$ & $\begin{array}{l}20: 56: 41.9 \\
44: 41: 13.5\end{array}$ & 12.56 & $\begin{array}{l}0.63 \\
0.45 \\
1.04\end{array}$ & $\begin{array}{l}0.48 \\
0.35 \\
0.87\end{array}$ & & A5 V & $\operatorname{lm}$ \\
\hline 38 & 6 & $\mathrm{~J} 20562218+443720$ & $\begin{array}{r}83.5 \\
-40.3\end{array}$ & $\begin{array}{l}20: 56: 22.1 \\
44: 37: 20.0\end{array}$ & 12.59 & $\begin{array}{l}0.52 \\
0.63 \\
1.06\end{array}$ & $\begin{array}{l}0.44 \\
0.55 \\
0.97\end{array}$ & & $\mathrm{~A} 3 \mathrm{~V}$ & $\operatorname{lm}$ \\
\hline 48 & 13 & $\mathrm{~J} 20562809+443501$ & $\begin{array}{r}18.7 \\
-180.9\end{array}$ & $\begin{array}{l}20: 56: 28.1 \\
44: 34: 58.9\end{array}$ & 13.19 & $\begin{array}{l}0.80 \\
0.43 \\
0.96\end{array}$ & $\begin{array}{l}0.75 \\
0.38\end{array}$ & & $\mathrm{~A} 2 \mathrm{~V}$ & $n m$ \\
\hline 53 & 28 & J20564026+443926 & $\begin{array}{r}-109.2 \\
86.6\end{array}$ & $\begin{array}{l}20: 56: 40.2 \\
44: 39: 26.1\end{array}$ & 13.44 & $\begin{array}{l}0.79 \\
0.66 \\
1.25\end{array}$ & $\begin{array}{l}0.71 \\
0.58 \\
1.16\end{array}$ & & A3 V & $p m$ \\
\hline
\end{tabular}

Notes:

- \# and \#ZS columns indicate our numbering and that from Zdanavicius \& Straizys (1990), respectively.

- \#Zs numbers with an asterisk indicate stars separated in two components (values correspond to the brightest one).

- The full Table 2 is available, with all the photometric measurements, in electronic form at the CDS, via anonymous ftp to cdsarc.u-strasbg.fr (130.79.128.5) or via http://cdsweb.u-strasbg.fr/cgi-bin/qcat?]/A+A/419/149 


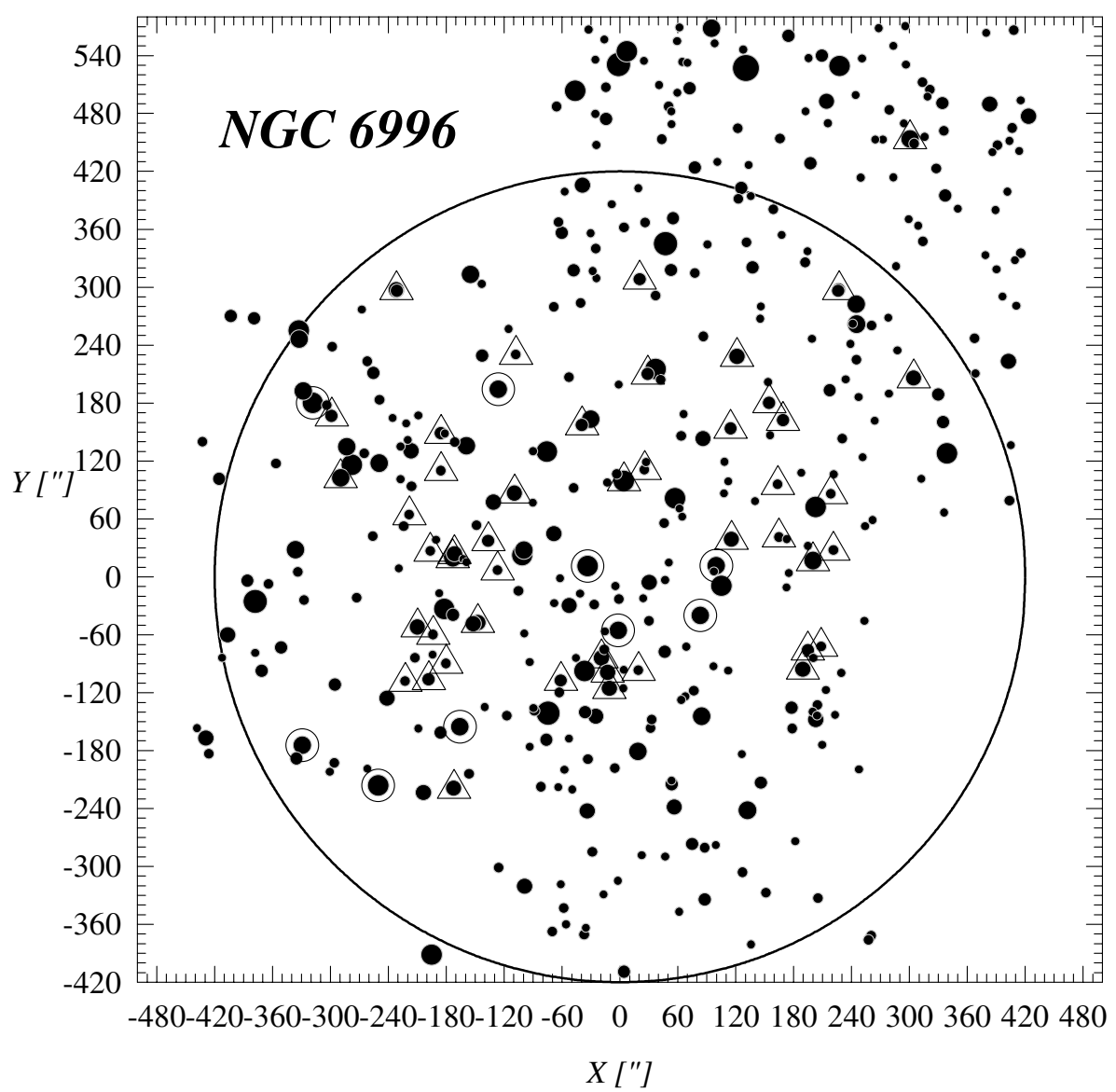

Fig. 3. Finding chart of the NGC 6996 region ( $V$ filter). The black solid circle indicates the adopted angular size $\left(\right.$ radius $\left.=7^{\prime}\right)$ for the cluster $($ see Sect. 3.1 and Fig. 5). Adopted likely and probable cluster members are enclosed in small circles and triangles, respectively. For a coordinate reference, the center $(X=0 ; Y=0)$ corresponds to the cluster coordinates (see Sect. 3.1) and each $X-Y$ are expressed in arcsecs. North is up, East to the left.

catalogue (Høg et al. 2000). With this aim in mind, we collected proper motion components for 22 stars in a field of $10^{\prime}$ radius centered on NGC 6996. They are shown as a vector point diagram in Fig. 6. The point distribution is characterized by a clump of several stars and few others placed around it. This can be readily interpreted as indicative of the presence of a star cluster. However, on the basis of the analysis here below, we found only 3 member stars with available proper motion from Tycho-2, and therefore we refrain from any kinematic analysis of NGC 6996 stars.

\subsection{Photometric diagrams}

The color-color diagrams (CCDs) and the color-magnitude diagrams (CMDs) from our data are shown in Figs. 7 and 8, respectively. In Fig. 9 we present the corresponding CMDs from 2MASS data for the cluster region stars $\left(R<7^{\prime}\right)$ and for a region around it (see caption) that is used as the comparison field. Some remarkable features in these diagrams are the following ones:

- The Main Sequence (MS) in Figs. 7-9 is significantly wide, a fact that we mainly ascribe to differential reddening due to the patchy distribution of gas and dust inside the cluster itself;
- the reddening (see Fig. 7b) law inside NGC 6996 does not seem normal, and turns out to be significantly different from that commonly accepted to hold for the Cygnus region (Johnson 1965). Indeed most of the stars follow a parallel path to a higher excess ratio. This is quite common in very young open clusters and star forming regions (e.g. Vázquez et al. 1996; Carraro et al. 2003) but less so in older clusters;

- a noticeable gap appears at $B-V \approx 0.6$ and $V \approx 13$. This is not an unexpected feature, since several other young or intermediate age clusters have been found to exhibit gaps (e.g. Giorgi et al. 2002; Yadav \& Sagar 2002; Baume et al. 2003b).

\subsection{Cluster membership}

As a first step, we base our membership assignment procedure on a synoptic analysis of the star positions in the various photometric diagrams (e.g. Baume 1999, 2003a,b; Carraro 2002). By inspecting Fig. $7 \mathrm{a}$, we notice that up to $(B-V) \approx 0.6$ stars are placed on an apparent easily recognizable MS composed of B and later type stars according to a Schmidt-Kaler's (1982) ZAMS. They have also a compatible position on the CMDs 


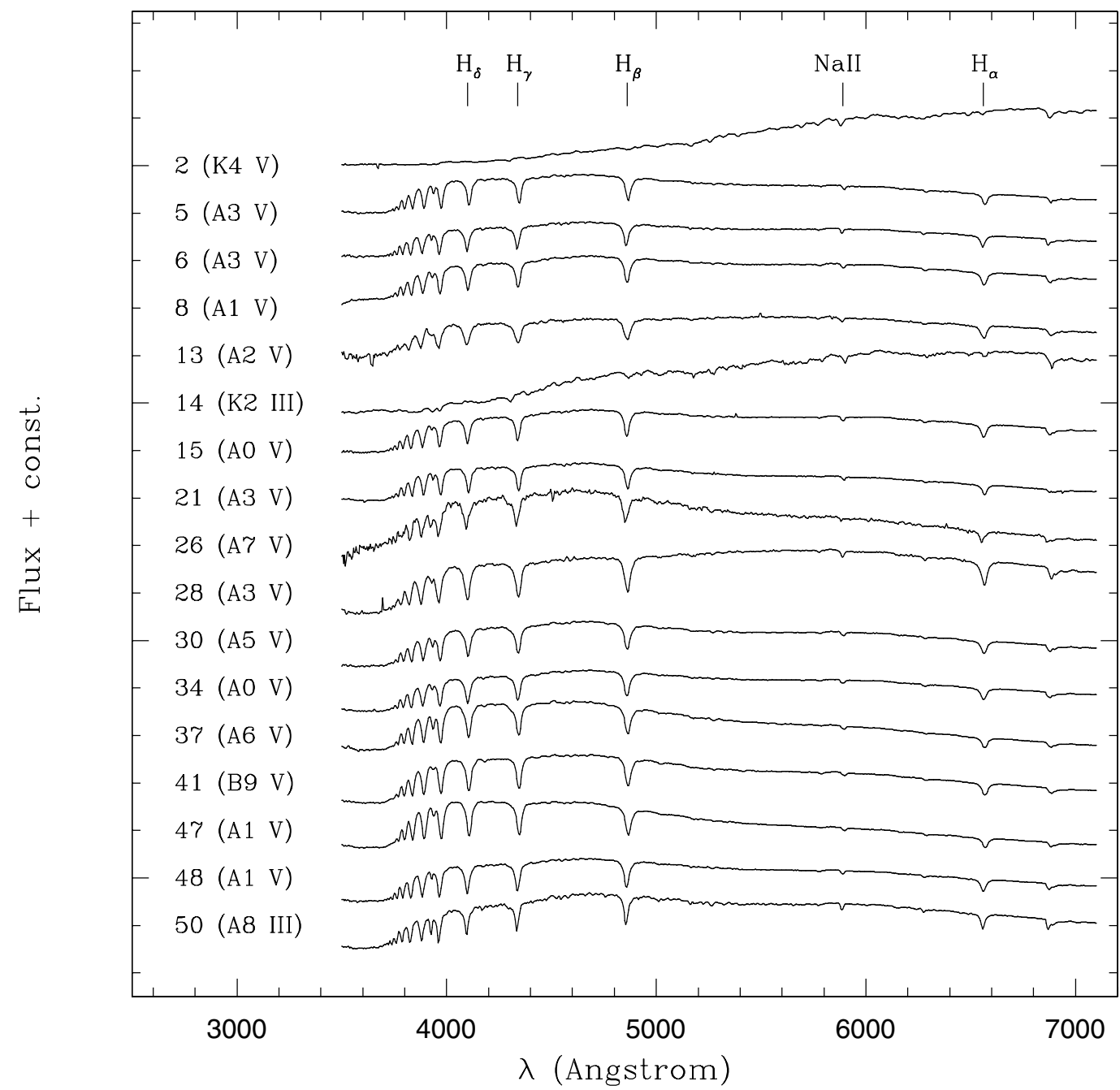

Fig. 4. Spectra of some bright stars in the field of NGC 6996. A few interesting lines are indicated. See Table 2 for details. Numbers correspond to the Zdanavicius \& Straizys (1990) identification.

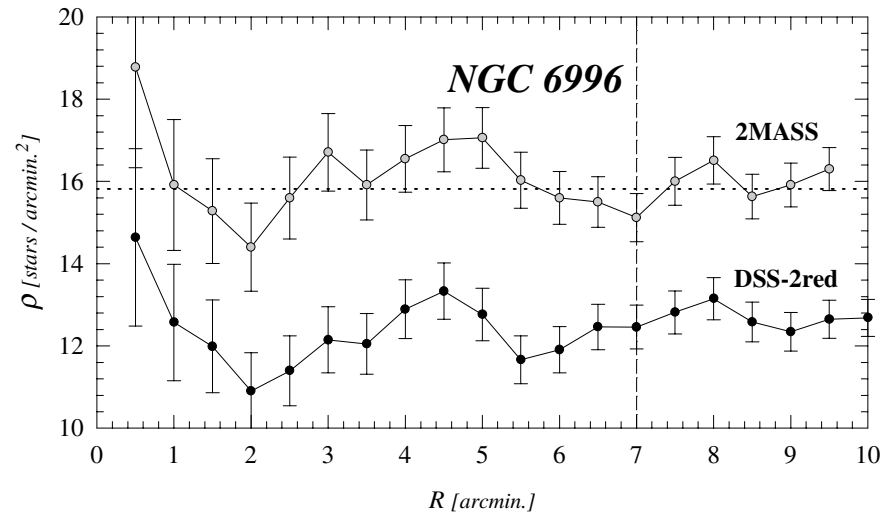

Fig. 5. Stellar density in the region of NGC 6996 as a function of the radius from 2MASS and DSS-2 red data. The dashed line indicates the adopted limit for the cluster.

of Figs. 8 and 9 down to $V \approx 13.5$. However, the considered B-type stars are more dispersed in these diagrams.

As a second step, we combine the magnitudes and colors with our spectral classification and that given by Zdanavicius \& Straizys (1990), when available, and the result is that most

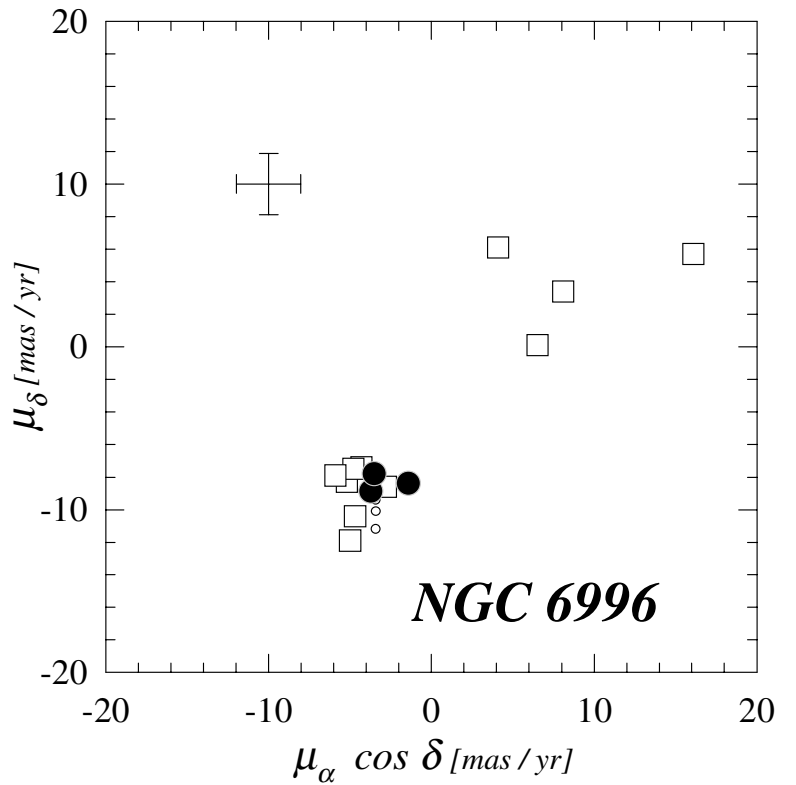

Fig. 6. Vector point diagram for the stars $10^{\prime}$ around the center of NGC 6996 (data from Tycho-2 catalogue). See Fig. 7 caption for the meaning of the symbols. The cross indicates the mean error values. 

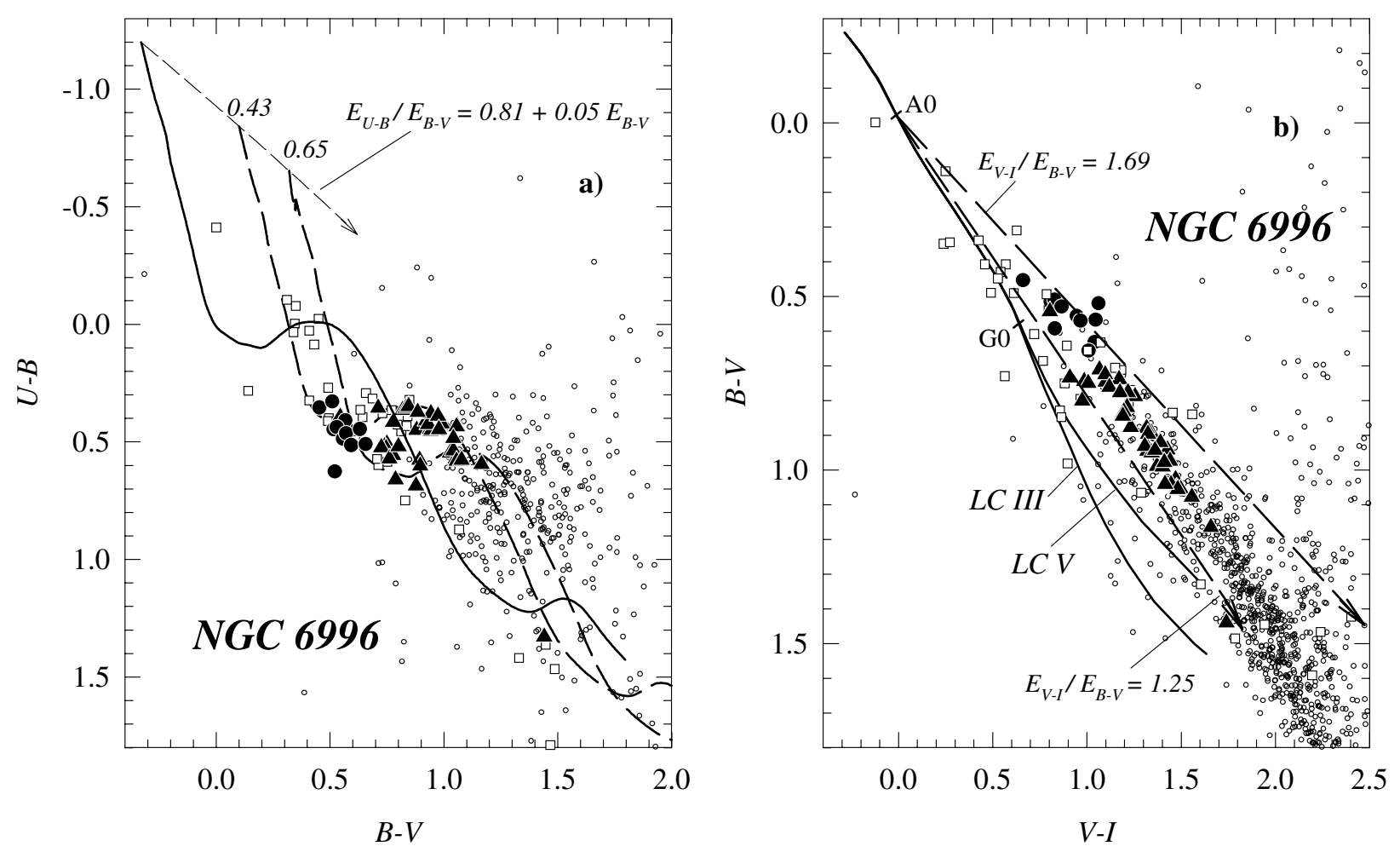

Fig. 7. Color-color diagrams (CCDs) of stars in the region of NGC 6996. a) $U-B$ vs. $B-V$ diagram. Symbols have the following meaning: circles are likely member stars $(\mathrm{lm})$, triangles are probable member stars $(\mathrm{pm})$, white squares are non-members $(\mathrm{nm})$ and small hollow circles are stars without any membership assignment. The solid line is the Schmidt-Kaler's (1982) ZAMS, whereas the dashed lines are the same ZAMS, but shifted by $E_{B-V}=0.43$ and 0.65 , respectively (see also Fig. 10a). The dashed arrow indicates the reddening path. b) $B-V$ vs. $V-I$ diagram. Symbols as in Fig. 7a. Solid lines are the intrinsic positions for stars of luminosity classes V and III (Cousins 1978a,b). The two dashed lines give the typical excess ratio for the Cygnus region $\left(E_{V-I} / E_{B-V}=1.25\right)$ and that adopted for the cluster $\left(E_{V-I} / E_{B-V}=1.69\right)$.

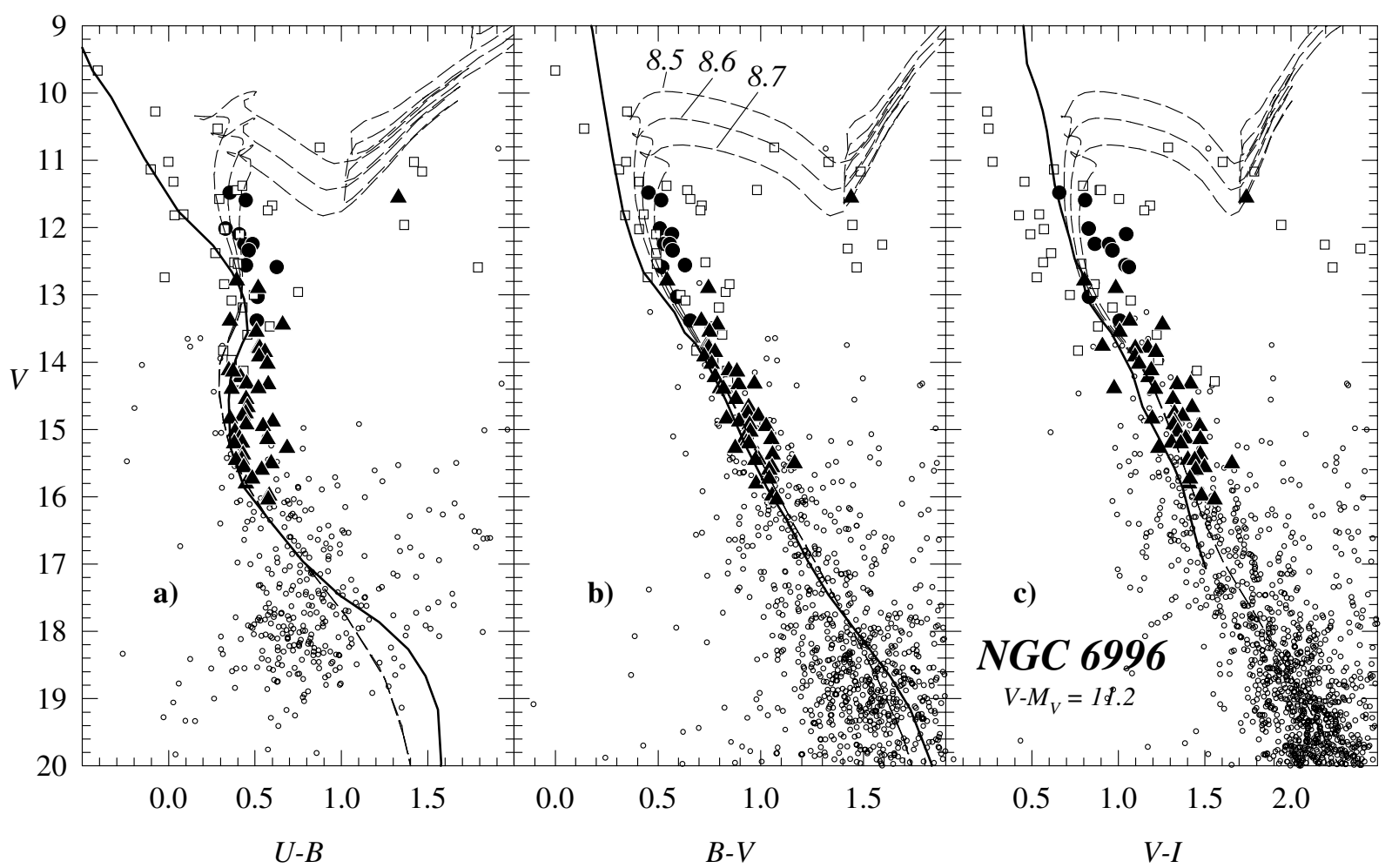

Fig. 8. Color-magnitude diagrams (CMDs) for all the stars covered in the field of NGC 6996. Symbols as in Fig. 7a. The solid line is the Schmidt-Kaler (1982) empirical ZAMS shifted by the apparent distance modulus $V-M_{V}=11.2\left(V-M_{V}=V_{0}-M_{V}+4.1 \times\left(E_{B-V}\right)\right.$, see Sect. 4).The dashed curves are the isochrones from Girardi et al. (2000). The reported numbers give the log (age). 


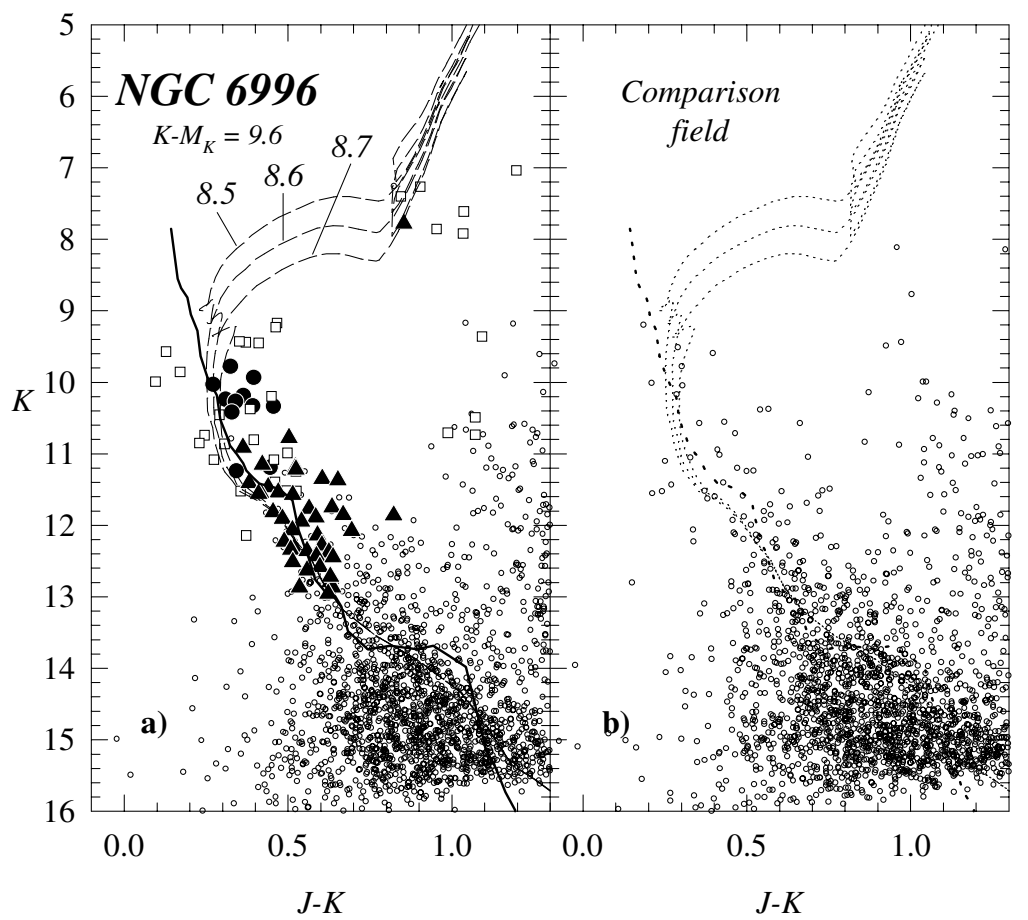

Fig. 9. CMDs from the 2MASS catalog. Symbols as in Fig. 7a. a) Stars placed inside the cluster area $\left(R<7^{\prime}\right.$, see Fig. 5). b) Stars located in the comparison field around the cluster $\left(R>8.85\right.$ and inside a box $\left.20^{\prime} \times 20^{\prime}\right)$. The solid line in panel a) and the dotted one in panel b) are the intrinsic position for MS stars from the Schmidt-Kaler (1982) and Koorneef (1983) calibrations fitted to the apparent distance modulus $K-M_{K}=9.6\left(K-M_{K}=V_{0}-M_{V}+(3.1-2.78)(4.1 / 3.1) E_{B-V}\right.$, see Sect. 4). The dashed curves are the isochrones from Girardi et al. (2000). The reported numbers give the log $($ age $)$.

of the brighter stars have very low excess values or/and low distance modulus, and only A-type stars have acceptable solutions. Therefore, the stars from the first group were considered as cluster non members ( $\mathrm{nm}$, probably interlopers), whereas the later ones as likely cluster members $(\mathrm{lm})$. Additionally, following the ZAMS path along the CCDs and CMDs toward laterthan-A spectral types, we identify other stars with compatible positions on the photometric diagrams. Here however the stellar contamination by field stars starts to become important, and therefore we adopt them as probable cluster members $(\mathrm{pm})$. This membership assignment produces a number of stars in different bins of $V$ magnitude that is in agreement with the overexcess of stars present in Fig. 9a when compared to Fig. 9b.

Stars \# ZS 6 and 14 (numbering from Zdanavicius \& Straizys 1990) deserve special attention. The former lies below the ZAMS in the CCD of Fig. 7a, and by inspecting also its position in the CMDs we interpret its color as due to binarity, the secondary being a cool red star. The latter is a red star classified as a K2 III. Looking at its location in the CMDs, also in relation to the superposed isochrones (see below), we are inclined to consider it a cluster member. Unfortunately, it has no proper motion measurement (from Tycho-2) and therefore we adopt it as a probable member $(\mathrm{pm})$.

\section{Cluster basic parameters}

\subsection{Corrected colors and magnitudes}

To infer the intrinsic colors, when spectral classification is available, we derive individual excesses by using
Schmidt-Kaler (1982) relations. Figures $7 \mathrm{a}$ and 10b show that the typical relation $E_{U-B} / E_{B-V}=0.81+0.05 E_{B-V}$ for the Cygnus region (Johnson 1965) fits the point distribution much better than the normal one $\left(E_{U-B} / E_{B-V}=0.72\right)$. We use then the former relation to obtain additional individual excesses from the CCD of Fig. 7a for stars without spectral classification. Then by considering the adopted likely members and probable members with $V<14$, we found a mean excesses of $E_{B-V}=0.52 \pm 0.08$ (s.d.) and $E_{U-B}=0.44 \pm 0.08$ (s.d.). The mean values are then adopted as representative of the cluster color excess, whilst the lowest value is interpreted as the foreground color excess.

The reddening law in the direction of NGC 6996 appears to be anomalous, and therefore we proceeded to compute the ratio $R_{V}=A_{V} / E_{B-V}$ by using the Excesses Relation Method (Fig. 10c), the Variable Extinction Method (Fig. 10d) and the Color Difference Method (Fig. 11). To build Figs. 10c and 10d, we compute individual $E_{B-V}, E_{V-I}$ and $V-M_{V}$ values by means of the Schmidt-Kaler (1982) and Cousins (1978a,b) calibration relations, a method also applied in Tr 14 (Vázquez et al. 1996) and in NGC 3293 (Baume et al. 2003). In the case of the Color Difference Method we combined the information from spectral classification, optical photometry and near infrared one from the 2MASS. For other applications of this method, see The \& Graafland (1995) and Carraro et al. (2003).

It is clear from Fig. 10c that star positions follow a path different from the typical one for Cygnus region $\left(E_{V-I} / E_{B-V}=\right.$ 1.25) resulting in a higher $R_{V}$ value. This is not evident in Fig. 10d where there is a larger spread due probably to the 


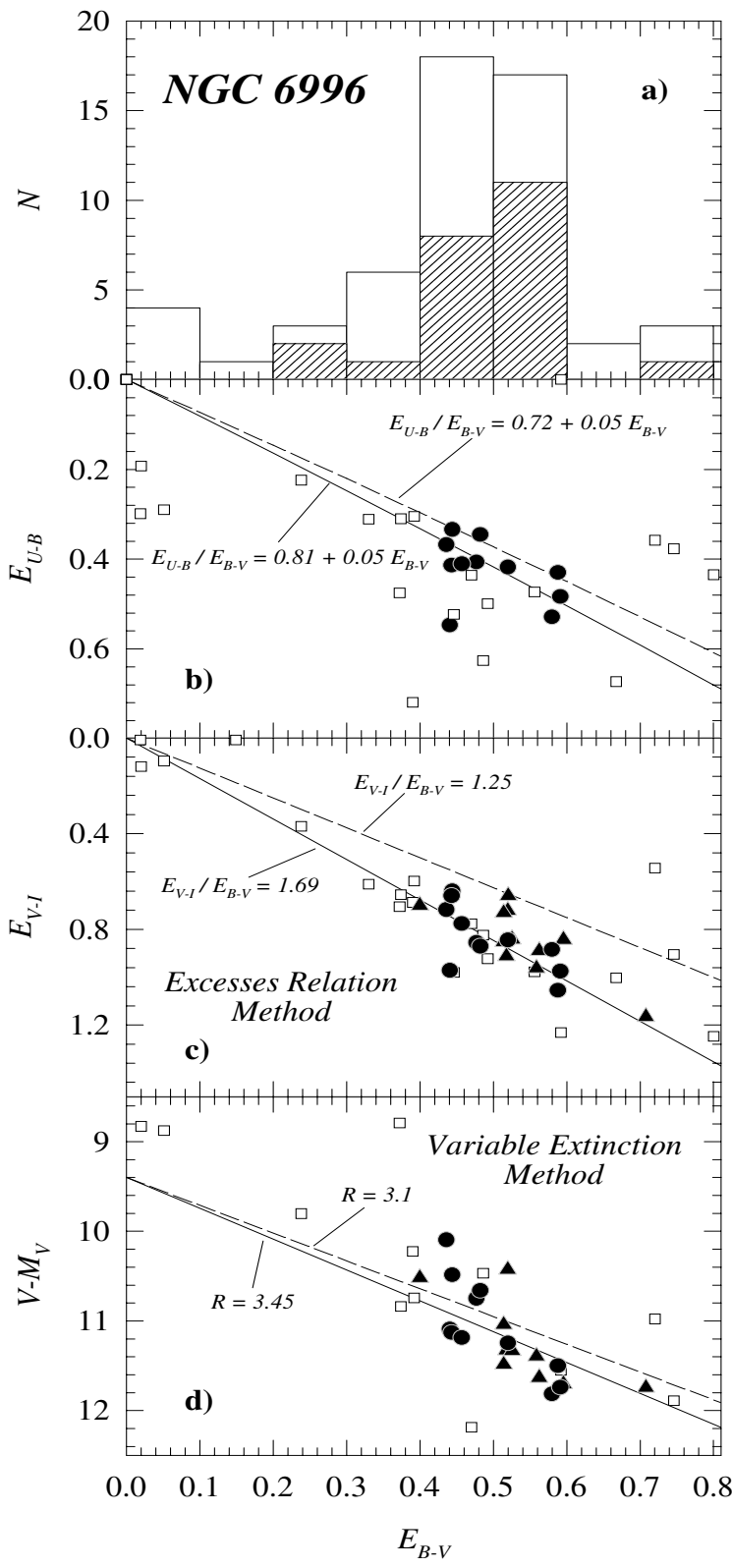

Fig. 10. a) $E_{B-V}$ distribution for stars inside the $7^{\prime}$ cluster radius (white histogram) and for adopted likely and probable cluster members (dashed histogram). b) $E_{U-B}$ vs. $E_{B-V}$ diagram for stars with available spectral classification. c) $E_{V-I}$ vs. $E_{B-V}$ diagram. d) Variable extinction method. Symbols in panels b), c) and d) as in Fig. 7a. Dashed lines represent normal relations and solid ones those obtained for NGC 6996.

presence of binaries. However, by performing least squares fittings over Figs. 10c and 10d and an extrapolation in Fig. 11, we obtain similar values (see Table 3 ). Therefore we adopt $R_{V}=4.1$ to compute corrected magnitudes $V_{0}=V-R_{V} \times E_{B-V}$ for cluster likely and probable members. This $R_{V}$ value yields a mean cluster absorption $\left\langle A_{V}\right\rangle=2.13$. It is worth noticing that this estimate is comparable with the absorption map in this region obtained by Goudis \& White (1979).
Table 3. Computed $R_{V}$ values by using different methods.

\begin{tabular}{ll}
\hline \hline Method & Obtained $R_{V}$ value \\
\hline Variable Extinction & $3.4 \pm 0.2$ \\
Excesses Relation & $4.2 \pm 0.1$ \\
Color Difference & $4.0 \pm 0.4($ s.d. $)$ \\
\hline
\end{tabular}

\subsection{Cluster distance and age}

The distance of NGC 6996 is derived by superposing the Schmidt-Kaler (1982) ZAMS onto the reddening-free CMD (Fig. 12). The best ZAMS fitting was achieved for a distance modulus $V_{0}-M_{V}=9.4 \pm 0.2$ (error from inspection). We also apply the spectroscopic parallax method to 10 likely and probable member stars of luminosity class V (see Table 3 ) by using the relation of spectral types and $M_{V}$ from Schmidt-Kaler (1982). This method yields a value $V_{0}-M_{V}=9.0 \pm 0.4$ (s.d.). The large dispersion of the last value and its difference from the ZAMS fit can be ascribed to the presence of binary and somewhat evolved stars included in the computation. The adopted distance modulus is therefore $V_{0}-M_{V}=9.4 \pm 0.2$ which in turn implies that NGC 6996 is located $760 \pm 70$ pc away from the Sun.

For the age of NGC 6996, we superimposed on the CMD (see Fig. 12) a set of isochrones derived from Girardi et al. (2000) evolutionary models (computed with solar metallicity, mass loss and overshooting). The fit wasperformed taking into account only MS stars and is compatible with an age for the cluster of about $310 \mathrm{Myr}(\log (8.5))$. By assuming that stars having A0 spectral type are still along the MS, we derive again an age near to $390 \mathrm{Myr}(\log$ (8.6)) for this cluster. Both procedures yield then similar results and we adopt $350 \pm 50 \mathrm{Myr}$ as the cluster age.

\section{Discussion and conclusion}

We have presented the first multicolor CCD photometric study in the region of the open cluster NGC 6996 together with spectral classification of some bright stars. NGC 6996 turns out to be a moderate-age open cluster $(\approx 350 \mathrm{Myr})$ located close to the west edge of NGC 7000, the North America Nebula. Our analysis places the cluster at a distance of $760 \pm 70 \mathrm{pc}$. Evidence emerges of a significant differential reddening affecting the stars' positions in all the photometric diagrams. We also point out that the reddening law has an anomalous value in the direction of this cluster.

To address the issue of its possible connection with the Nebula, we first summarize what it is known about the distance of the Nebula itself (see for reference Fig. 1). According to Straizys et al. (1999) the dust cloud that separates the North America and Pelican nebulae is placed at about $580 \mathrm{pc}$ form the Sun. For the HII region (NGC 7000 itself), the distance is far from being reasonably contrained. Different works claim for very different distance estimates, ranging from $420 \mathrm{pc}$ (Beer 1964) to 1980 pc (Dieter 1967). HD 199579, an O6 star considered responsible for at least part of the excitation of the region, is placed at a distance of $1200 \mathrm{pc}$ by Miller (1968) and 830 pc by Garmany \& Stencel (1992), who consider the star a 


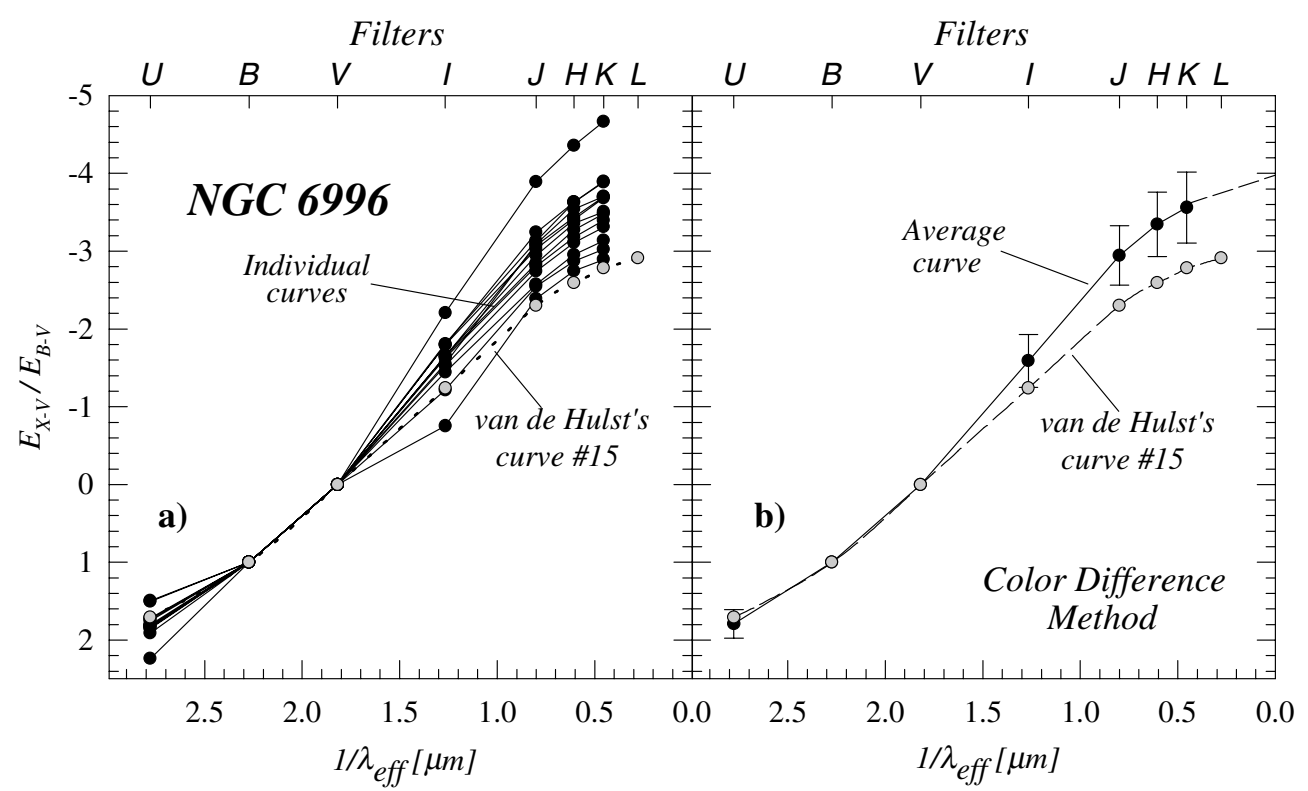

Fig. 11. The color difference method. a) Solid lines are individual curves computed for stars with spectral classification and luminosity class $V$ (see Table 2). b) Solid line is the obtained average curve together with their extrapolation up to $1 / \lambda_{\text {eff }}=0$. Dotted lines with grey simbols in both panels are the extinction curve \# 15 from van de Hulst (Johnson 1968).

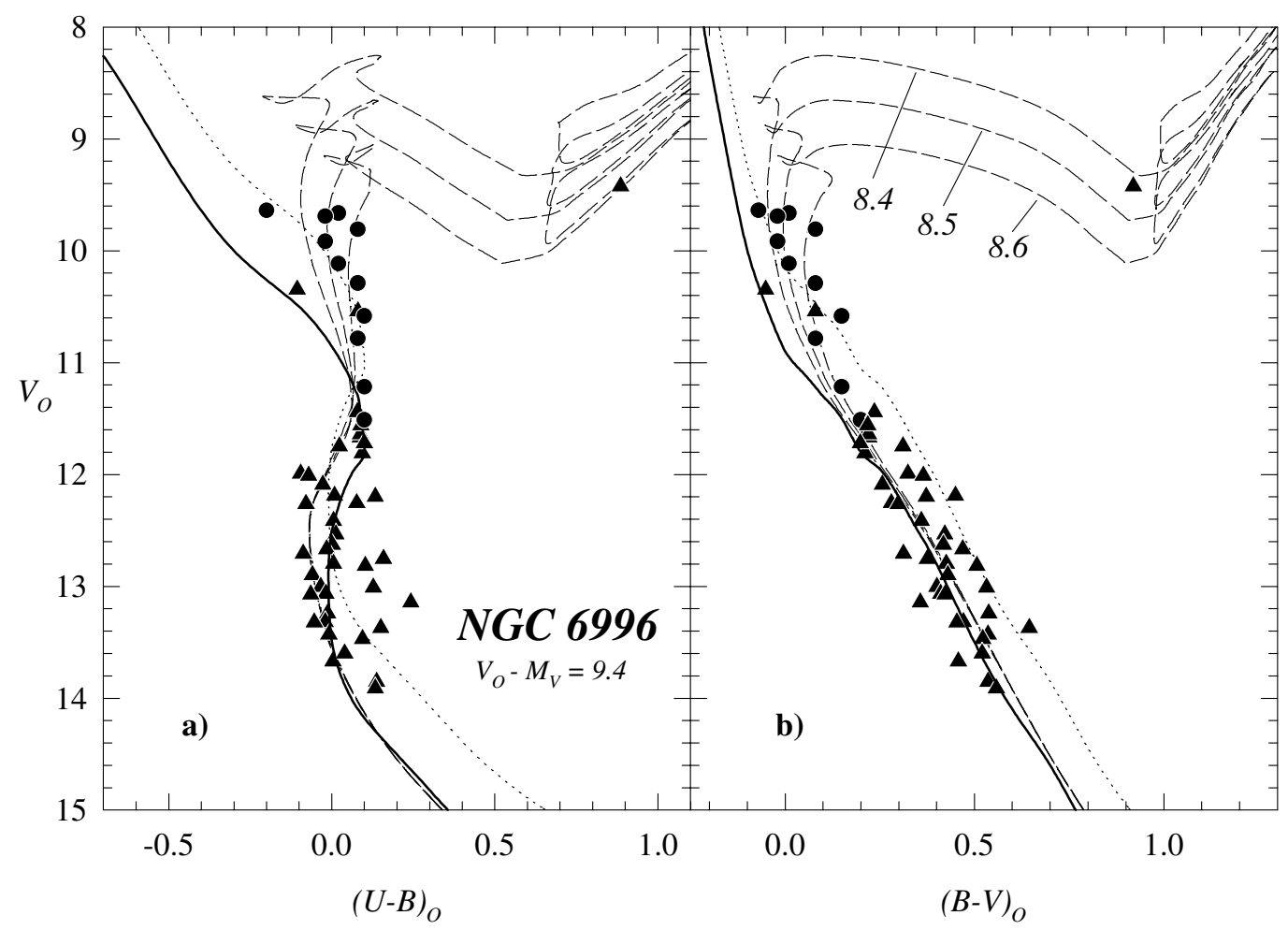

Fig. 12. $V_{0}$ vs. $(B-V)_{0}$ diagram of the likely and probable member stars in the region of NGC 6996. Symbols as in Fig. 7a. Solid and dotted lines are the Schmidt-Kaler (1982) ZAMS shifted by the adopted distance modulus $V_{0}-M_{V}=9.4$ and the 0.75 envelope limit for binaries. Dashed curves are the isochrones from Girardi et al. (2000). Numbers give the log (age).

member of the Cyg OB7 association. According to many other authors however, the most accepted value seems to be $\sim 1 \mathrm{kpc}$ (Downes \& Rinehart 1966; Wendeker 1968; Goudis 1976; Bally \& Scoville 1980).

If we accept this value as the distance of NGC 7000, NGC 6996 turns out to have no apparent relationship to the
HII region, the cluster being placed about $300 \mathrm{pc}$ closer to the Sun. The cluster age and the absence of early spectral type stars further corroborate this hypotesis.

The computed $R_{V}$ value for the cluster region is higher than the common one holding for the Galaxy (3.1, Mathis 1990), and is at odds with previous studies (e.g. Cambrésy et al. 2002) 
which assign to NGC 7000 a normal $R_{V}$ ratio. High $R_{V}$ values are indicative of the presence of dust grain of large size, typically larger than 0.05 micron. Since NGC 6996 is dominated by A type stars, UV radiation is not very effective, and one expects that dust grains grow in size (Kim \& Martin 1996). Another possibility would be that small size dust grains have been kicked off the cluster by a presumed population of massive stars that already have died as type II SNæ (McKee 1989), a scenario which is quite compatible with the age of the cluster.

Acknowledgements. The authors acknowledge the Asiago Observatory staff for the technical support and the director of Teramo Observatory for the generous time allocation. The work of G.B. is supported by the Università di Padova (Italy) through a postdoctoral grant.

\section{References}

Baume, G., Vázquez, R. A., Carraro, G., \& Feinstein, A. 2003a, A\&A, 402, 549

Baume, G., Moitinho, A., Giorgi, E. E., Carraro, G., \& Vázquez, R. A. 2003b, A\&A, in press

Bally, J., \& Scoville, N. Z. 1980, ApJ, 239, 121

Barkhatova, K. A. 1958, Astron. Zh., 35, 448

Beer, A. 1964, MNRAS, 128, 261

Cambrésy, L., Beichman, C. A., Jarrett, T. H., \& Cutri, R. M. 2002, AJ, 123, 2559

Carraro, G. 2002, MNRAS, 331, 785

Carraro, G., Romaniello, M., Ventura, P., \& Patat, F. 2003, A\&A, in press

Cousins, A. W. J. 1978a, MNSSA, 37, 62

Cousins, A. W. J. 1978b, MNSSA, 37, 77

Desidera, S., Fantinel, D., \& Giro, E. 2002, AFOSC user manual

Dias, W. S., Alessi, B. S., Moitinho, A., \& Lepine, J. R. D. 2002, A\&A, 389, 871

Dieter, M. 1967, ApJ, 150, 435
Downes, D., \& Reinhart, R. 1966, ApJ, 144, 937

Garmany, C. D., \& Stencel, R. E. 1992, A\&AS, 94, 211

Giorgi, E. E., Vázquez, R. A., Baume, G., Seggewiss, W., \& Will, J.-M. 2002, A\&A, 381, 884

Girardi, L., Bressan, A., Bertelli, G., \& Chiosi, C. 2000, A\&AS, 141, 371

Goudis, C. 1976, Ap. Space. Sci., 39, 173

Goudis, C., \& White, N. J. 1979, A\&A, 78, 373

Høg, E., Fabricius, C., Makarov, V. V., et al. 2000, A\&A, 357, 367

Jacoby, G. H., Hunter, D. H., \& Christian, C. A. 1984, ApJS, 56, 257

Jaschek, C., \& Jaschek, M. 1987, The classification of stars (Cambridge University Press)

Johnson, H. L. 1965, ApJ, 141, 923

Kim, S. H., \& Martin, P. G. 1996, ApJ, 462, 296

Koornneef, J. 1983, A\&A, 128, 84

Landolt, A. U. 1992, AJ, 104, 340

Lyngå, G. 1987, Catalog of Open Star Cluster Data, Strasbourg, CDS

Mathis, J. S. 1990, ARA\&A, 28, 37

McKee, C. 1989, IAU, 135, 431

Miller, J. S. 1968, ApJ, 151, 473

Müller, H. 1936, Veröff. Astron. Rech. Inst. Berlin, No. 53

Schmidt-Kaler, Th. 1982, Landolt-Börnstein, Numerical data and Functional Relationships in Science and Technology, New Series, Group VI, 2(b), ed. K. Schaifers, \& H. H. Voigt (Berlin: Springer Verlag), 14

Stetson, P. B. 1987, PASP, 99, 191

Straizys, V., Corbally, C. J., \& Laugalys, V. 1999, BaltA, 8, 355

Subramanian, A., Gorti, U., Sagar, R., \& Bhatt, H. C. 1985, A\&A, 302,86

Thé, P. S., \& Graafland, F. 1995, Rev. Mex. Astron. Astrof., 2, 75

Torres-Dodgen, A. V., \& Weaver, W. B. 1993, PASP, 105, 693

Vázquez, R. A., Baume, G., Feinstein, A., \& Prado, P. 1996, A\&A, 116,75

Wendeker, H. 1968, Za. f. Ap., 68, 368

Yadav, R. K. S., \& Sagar, R. 2002, MNRAS, 337, 133

Zdanavicius, K., \& Straizys, V. 1990, Ap\&SS, 173, 309 